Review of Dense Plasma Focus

Technology for Intense and Directional Neutron Sources

Vincent Tang, Brian Rusnak

February 29, 2008 
This document was prepared as an account of work sponsored by an agency of the United States government. Neither the United States government nor Lawrence Livermore National Security, LLC, nor any of their employees makes any warranty, expressed or implied, or assumes any legal liability or responsibility for the accuracy, completeness, or usefulness of any information, apparatus, product, or process disclosed, or represents that its use would not infringe privately owned rights. Reference herein to any specific commercial product, process, or service by trade name, trademark, manufacturer, or otherwise does not necessarily constitute or imply its endorsement, recommendation, or favoring by the United States government or Lawrence Livermore National Security, LLC. The views and opinions of authors expressed herein do not necessarily state or reflect those of the United States government or Lawrence Livermore National Security, LLC, and shall not be used for advertising or product endorsement purposes.

This work performed under the auspices of the U.S. Department of Energy by Lawrence Livermore National Laboratory under Contract DE-AC52-07NA27344. 


\section{Review of Dense Plasma Focus Technology for Intense and Directional Neutron Sources}

Feb. 25, 2008

Vincent Tang, National Security Engineering Division, x2-0126, tang23@1lnl.gov

Brian Rusnak, N Division, x2-0435, rusnak1@1ln1.gov

\section{Abstract}

We believe the Dense Plasma Focus (DPF) has possible applications as a unique high intensity neutron source when compared with conventional accelerator-driven neutron generators or ${ }^{252} \mathrm{Cf}$ isotope-based sources. We see two possible opportunities where DPF devices could have a major impact as an alternate radiological source in comparison with conventional technology in terms of average and especially peak neutron output, and also directional neutron emission via $\sim 100 \mathrm{MV} / \mathrm{m}$ plasma-based acceleration gradients. Here, we briefly review DPF technology and compare it to conventional neutron generators, and present our two research prospects.

\section{Device Description}

The DPF is a compact plasma device comprised primarily of a coaxial head or gun for generating a unique class of intense z-pinch plasmas. A moderate to high-power pulse forming network (PFN) initiates the plasma and drives the pinch effect. The coaxial gun itself is physically small; the electrodes typically have sizes on the order of $\sim 10 \mathrm{~cm}$ and the total size of the system is normally dominated by the PFN. While physically and operationally simple, these devices have been studied for over $\sim 40$ years worldwide as possible fusion reactors and intense neutron machines, and yet have still to be fully understood and characterized. The DPF has been shown to be a copious source of pulsed x-rays, ion and electron beams, and neutrons [1-32]. Acceleration gradients of $\sim 100 \mathrm{MV} / \mathrm{m}$ due to non-linear processes have also been observed in these plasmas. The physics behind the rather remarkable intensities and beam energies obtained in the pinch remains obtuse. Although the mechanisms of plasma formation, beam creation, and neutron production are not completely understood, significant amount of empirical, experimental, and modeling information is available to guide the design of DPF neutron sources [e.g. 33-35]. Table 1 summarizes the parameters of several DPF devices compared to some conventional accelerator and isotope based neutron sources and shows that they are not only competitive, but in some cases superior in their output.

In typical DPF operation, illustrated in Figure 1, plasma current sheets in a coaxial configuration or gun are first formed from flashover breakdown along insulator surfaces on the inner electrode and accelerated forward through $\mathbf{J} \times \mathbf{B}$ forces. A fill gas at the Torr level is normally used. At the end of the coaxial gun, the sheets expand and collide to form a $\sim \mathrm{cm}$ long high density $\left(\mathrm{n}_{\mathrm{e}} \sim 10^{19}-10^{20} / \mathrm{cc}, \mathrm{T}_{\mathrm{e}} \sim 1-10 \mathrm{keV}\right)$ z-pinch plasma on the tip of the inner electrode. In this region, the z-pinch is typically confined for $\sim 100 \mathrm{~ns}$ and intense $\mathrm{x}$-rays, ion, and electron beams are generated through a complex combination of non-linear instabilities and other mechanisms that result in effective acceleration gradients of $\sim 100 \mathrm{MV} / \mathrm{m}$. Axial ion beams from the anode with energies in the hundreds of $\mathrm{keV}$ to $\mathrm{MeV}$ range have been observed in numerous experiments [9$12,15-16,18,22-24,26]$, especially during low pressure operation. Abundant high-energy neutrons can be emitted through thermonuclear, beam-gas, and beam-beam fusion reactions in the pinch if deuterium and/or tritium is used as the filling gas $[1-6,9,14,18,24-29,31]$. Depending on the device and operating parameters, the axial neutron emission can be up to four 
times greater than the radial emission $[1,4,25]$. The D-D neutron output from $10^{4}$ to $10^{12}$ per $\sim 100 \mathrm{~ns}$ pulse, corresponding to peak neutron outputs of $\sim 10^{11}$ to $10^{19} / \mathrm{s}$, was found to conservatively scale with the pinch current to the fourth power in a range of devices powered by PFNs typically involving high voltage capacitors delivering $\sim 100 \mathrm{~J}$ to $\sim \mathrm{MJ}$.

The scaling basis is shown in Figure 1. The emitted D-D neutron energy spectra can have a wide spread; for example, energies up to $\sim 4 \mathrm{MeV}$ for axially forward neutrons were detected in a $27 \mathrm{~kJ}$ device [5].

\begin{tabular}{|c|c|c|c|c|c|c|}
\hline Device & $\begin{array}{l}\text { Average D-D n } \\
\text { output }(n / s)\end{array}$ & $\begin{array}{l}\text { Peak D-D } \\
\text { n output }(\mathrm{n} / \mathrm{s})\end{array}$ & $\begin{array}{l}\text { Rep rate } \\
(\mathrm{Hz})\end{array}$ & $\begin{array}{l}\text { Max neutron } \\
\text { energy }^{1}(\mathrm{MeV})\end{array}$ & $\begin{array}{l}\text { Neutron } \\
\text { Directionality }\end{array}$ & $\begin{array}{l}\text { Size w/PFN or } \\
\text { equivalent }\end{array}$ \\
\hline 125 J DPF [27] & $\sim>10^{6}$ & $2 \times 10^{13}(\sim 50 \mathrm{~ns})$ & $>1.0^{*}$ & $\sim 2.5-3$ & $\begin{array}{l}1 \text { to } 4 \mathrm{x} \\
\text { axial } \text { radial }^{2}\end{array}$ & $\begin{array}{l}\sim \text { portable tool } \\
\text { chest }\end{array}$ \\
\hline 6 kJ DPF [28] & $3 \times 10^{8}$ & $3 \times 10^{15}(\sim 100 \mathrm{~ns})$ & $\sim 1$ & $\sim 3-4$ & $\begin{array}{l}1 \text { to } 4 \mathrm{x} \\
\text { axial } / \text { radial }^{2}\end{array}$ & $\begin{array}{l}\text { large tool } \\
\text { chest }\end{array}$ \\
\hline 126 kJ DPF [2] & $2 \times 10^{10}-2 \times 10^{11}$ & $2 \times 10^{18}(\sim 100 \mathrm{~ns})$ & $\sim 0.1-1.0^{*}$ & $\sim 5-6$ & $\begin{array}{l}1 \text { to } 4 \mathrm{x} \\
{\text { axial } / \text { radial }^{2}}^{2}\end{array}$ & $\sim$ Small office* \\
\hline RFQ & $\sim 10^{10}$ & $10^{12}(\sim 100 \mu \mathrm{s})$ & 100 & $\sim 7$ & $\sim 45$ deg Cone & $\sim$ Small office \\
\hline $\begin{array}{l}\text { Neutron tube } \mathrm{w} / \\
\text { Penning source }\end{array}$ & $\sim 10^{6}$ & $\sim 10^{9}(\sim 10 \mu \mathrm{s})$ & 100 & $\sim 2.5-2.8$ & Little & $\begin{array}{l}\text { large tool } \\
\text { chest }\end{array}$ \\
\hline${ }^{252} \mathrm{Cf}$ & $\sim 10^{6}$ to $\sim 10^{9}(\mathrm{SF})$ & na & na & $\sim 5$ & None & $<$ palm \\
\hline
\end{tabular}
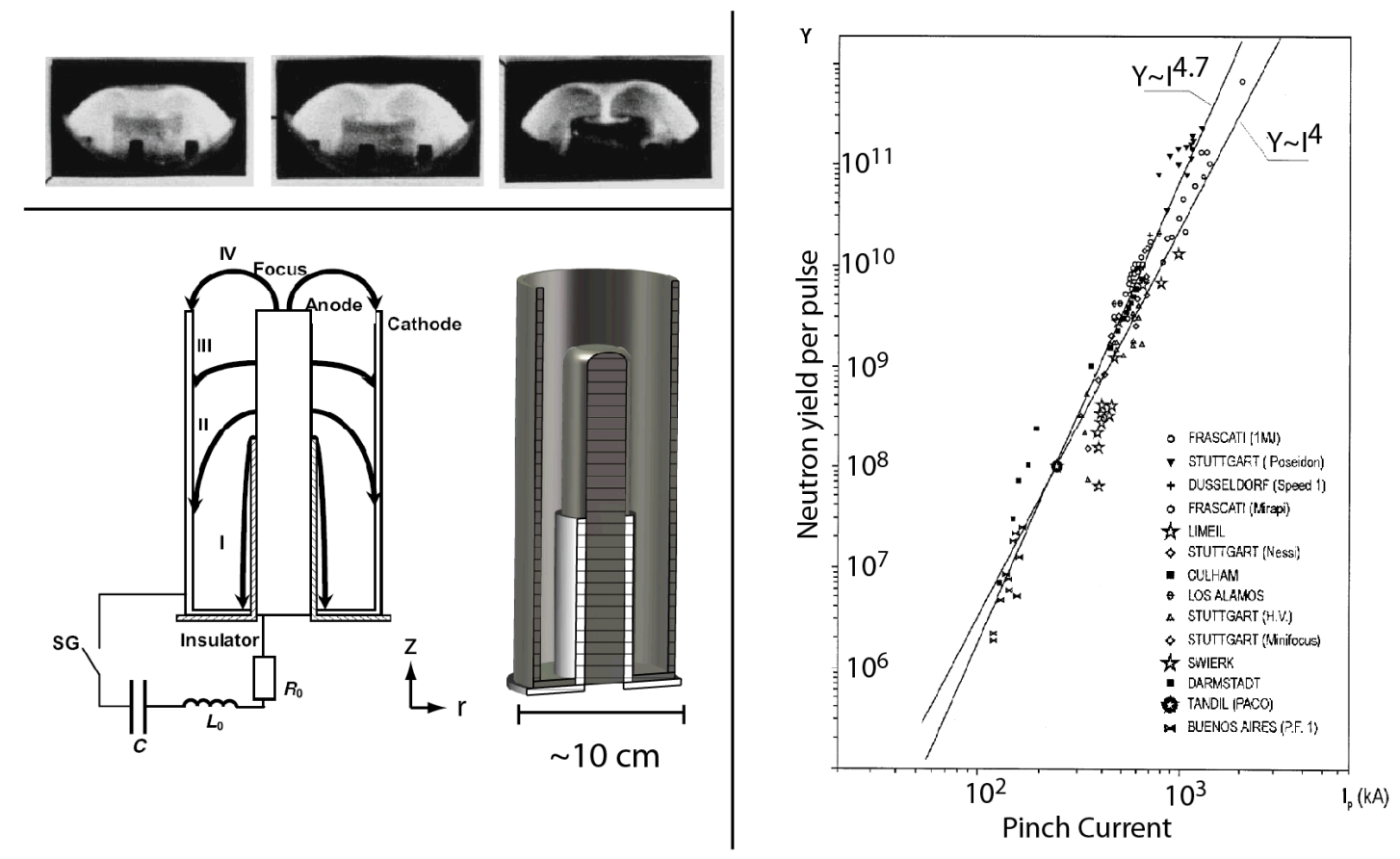

Figure 1 Left) Schematic of a dense plasma focus and its mode of operation. For reference, the axial direction refers to the z-axis. The photographs on top show the formation of the plasma from flashover of the insulator (I), followed by the sheet propagation (II, III) through collapse of the current sheets to form the pinch (IV). Right) Scaling of neutron yield per pulse for the D-D reaction as a function of DPF pinch current. [1, Pouzo, Soto]

\section{As An Alternative Neutron Source}

Straightforward engineering development of the DPF for field application would readily lead to a mobile DPF neutron source with significantly higher peak and average neutron output (and energy) compared with more conventional accelerator-driven sources. Recent work in Italy [28] has already demonstrated a semi-mobile deuterium $6 \mathrm{~kJ}$ plasma focus operating at $3 \times 10^{8} \mathrm{n} / \mathrm{s}$ using a repetition rate of $1 \mathrm{~Hz}$, giving a peak neutron output exceeding $\sim 10^{15} \mathrm{n} / \mathrm{s}$. Hundreds of 
successful shots per day were performed to test the reliability and repeatability of this device. The authors believe that given the research-oriented design of their PFN, conventional optimization of it for mobility would make their device readily portable. Another candidate is the significantly more compact $125 \mathrm{~J}$ device chargeable by a tractor battery [27] which demonstrated output up to $\sim 10^{6} \mathrm{n}$ per $50 \mathrm{~ns}$ pulse giving a peak neutron intensity of $\sim 2 \times 10^{13} \mathrm{n} / \mathrm{s}$. The neutron output of this smaller device is favorably above the neutron-pinch current scaling law. Existing DPF machines with their PFN have thus shown average neutron outputs up to a two orders of magnitude greater than conventional neutron generators based on Penning sources of approximately the same size, and peak neutron output up to six orders of magnitude larger. This is partly because the non-linear beam acceleration in the pinch region leads to higher energy ions which are much closer to the peak of the $\mathrm{D}(\mathrm{d}, \mathrm{n})$ cross section. Also, as mentioned, the neutron output can be axially favored. Compared with isotope sources of similar output, the advantages of the DPF are compelling in terms of possibly reduced shielding, source radioactivity, and pulsing capability. We therefore believe that an optimized mobile DPF source based on demonstrated experimental results could meet or exceed the performance of available source technologies, especially in interrogation schemes that favor extremely short pulses or high peak and average neutron output. If the device could be optimized to deliver greater currents of high energy ions, larger semi-mobile DPFs at $\sim 10^{11} \mathrm{n} / \mathrm{s}$ output could be favorable for various schemes, such as significantly shortening irradiation time for cargo interrogation.

\section{As An Advanced Accelerator Technology}

Another intriguing opportunity is the possibility of optimizing axial beam production in a DPF for providing MeV beams for a compact directional neutron source and other applications. This work would attempt to better understand and take advantage of the DPF's demonstrated $\sim 100 \mathrm{MV} / \mathrm{m}$ acceleration gradients. Generating higher energy ions allows more directional neutrons to be created via the kinematics of the D-D reaction, or through threshold kinematic reactions like p-Li if a near mono-energetic proton beam can be produced just above threshold energies. The majority of work done so far has been primarily for neutron optimization in the pinch, not necessarily ion beam production and acceleration. Experimentally, on smaller devices with capacitor energies in the $\mathrm{kJ}$ regime, the measured axial ions have energies in the low hundreds of $\mathrm{keV}$ range $[11,24,26]$. On larger devices with capacitor energies in the $10-100 \mathrm{~kJ}$ range, such as the Livermore DPF, $\sim 10^{14}$ ions with energies greater than $330 \mathrm{keV}$ were detected for each pulse. Roughly $10^{12}$ of those ions had energies greater than $5 \mathrm{MeV}$. These measured ion beam data for the Livermore device and the device configuration are shown in Figure 2.
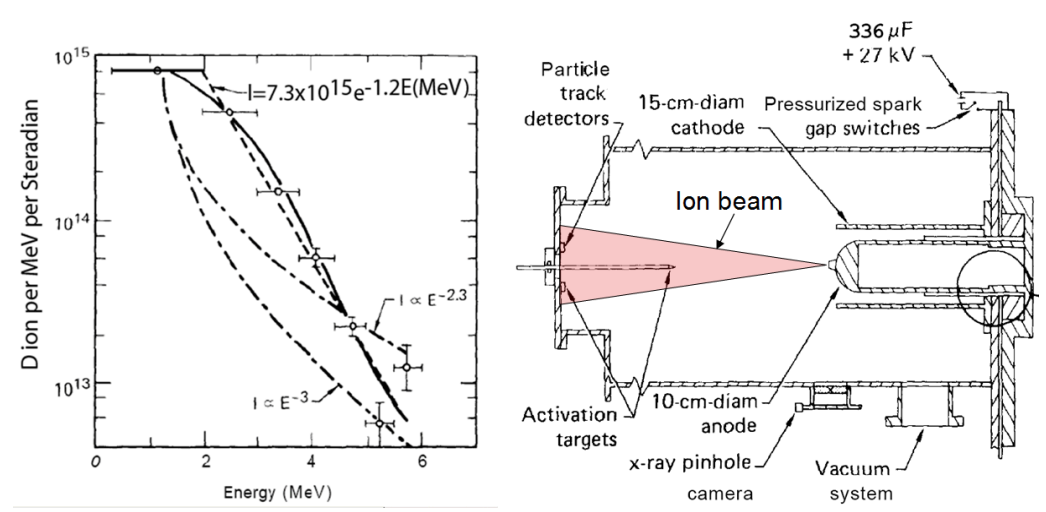

Figure 2) Axial beam spectrum from the Livermore DPF and the associated experimental geometry [10]. The device operated in the late seventies. [2] 
Incorporating a deuterated target into the Livermore device to better utilize these $\mathrm{MeV}$ level deuterons could have resulted in an additional $\sim 10^{7}-10^{8}$ forward directed neutrons per pulse. For this ion beam optimization approach, we propose first modeling and understanding in detail the DPF formation, beam creation, acceleration and collisional processes in order to numerically optimize the DPF for $\mathrm{MeV}$ level axial beam production and to examine if and how more qausi-mono-energetic beams can be created. Modeling could be approached through a linked series of circuit, magneto-hydrodynamic and particle-in-cell simulations [32, 36-42] to provide insight into the inner workings of the pinch which could not be performed in detail 20-30 years ago due to computational resource limitations. This simulation approach combined with today's computational power would allow us to study more complex scenarios, such as stacking of multiple DPFs with hollow electrodes for a multi-stage plasma based accelerator. If significant amount of $\mathrm{MeV}$ level beams could be produced from a DPF at the $\sim \mathrm{kJ}$ power supply level, a truly ultra-compact and portable directional neutron source should be possible. In addition to compact directional neutron production, remarkable opportunities for accelerator technology exist if the DPF can be used as the basis for a $\sim 100 \mathrm{MV} / \mathrm{m}$ plasma accelerator.

\section{Conclusion}

The DPF thus holds significant promise for compact neutron sources compared with conventional technology. Mobile sources with peak neutron outputs exceeding $\sim 10^{15} \mathrm{n} / \mathrm{s}$ should be feasible with some engineering development. Successful optimization of the DPF for axial beam production could allow intense directional neutron sources utilizing plasma-based accelerating gradients of $\sim 100 \mathrm{MV} / \mathrm{m}$.

\section{References}

[1] See J. W. Mather, "Investigation of the High Energy Acceleration Mode in the Coaxial Gun”, Phys. Fluids, S28 (1964) and J. W. Mather, "Formation of a High-Density Deuterium Plasma Focus", Phys. Fluids, 8, 2, 366 (1965) for the original work.

Some review papers are:

G. Decker and R. Wienecke, "Plasma Focus Devices", Physica, 82C, 155 (1976).

A. Bernard, P. Cloth, H. Conrads et al., "The Dense Plasma Focus-A High Intensity Neutron Source", Nuc. Instr. Meths. 145, 191 (1977).

J. Pouzo and M. Milanese, "Applications of the Dense Plasma Focus to Nuclear Fusion and Plasma Astrophysics", IEEE Plasma Sci., 31, 6, 1237, (2003).

L. Soto, "New trends and future perspectives on plasma focus research", Plasma Phys. Cont. Fusion., 47, A361 (2005).

[2] Previous LLNL DPF: T. Wainwright, W. Pickles, J. McClure, D. Price, and P. Eltgroth, "Results of the LLNL Plasma Focus Project", UCID-19175, technical report (1981).

[3] M. J. Bernstein, D. A. Meskan, and van Passen, "Space, Time, and Energy Distributions of Neutrons and X Rays from a Focused Plasma Discharge", Phys. Fluids, 12, 10, (1969).

[4] C. Patou, A. Simonnet, and J. Watteau, "Measured Anisotropies of the Plasma Focus Neutron Emission Compared with Proposed Mechanisms", Phys. Lett., 29A, 1, 1, (1969).

[5] M. J. Bernstein, "Deuteron Acceleration and Neutron Production in Pinch Discharges", Phys. Rev. Lett., 24, 13, (1970). Also, M. J. Bernstein, "Evidence for Nonthermonuclear neutron production in a plasma focus discharge", Phy. Lett., 31A, 6, 317, (1970).

[6] J. Lee, L. Shomo, M. Williams, and H. Hermansdorfer, "Neutron Production Mechanism in a Plasma Focus", Phys. Fluids, 14, 10, 2217, (1971). 
[7] H. Conrads, P. Cloth, M. Demmeler, and R. Hecker, "Velocity Distribution of the Ions Producing Neutrons in a Plasma Focus", Phys. Fluids, 15, 1, 209, (1972).

[8] S. P. Gary, "Ion kinematics in a plasma focus", Phys. Fluids, 16, 7, (1973). Also S. P. Gary, "Ion acceleration in a plasma focus", Phys. Fluids, 17, 11, (1974).

[9] H. Krompholz, L. Michel, K. Schonback, and H. Fischer, "Neutron-, Ion-, and Electron-Energy Spectra in a 1 kJ Plasma Focus”, Appl. Phys. 13, 29 (1977).

[10] R. Gullickson, W. Pickles, D. Price, H. Sahlin, and T. Wainwright, "Ion Beam Production in the Plasma Focus Device", UCRL-81962, LLNL preprint (1979).

[11] L. Bertalot, H. Herold, U. Jager et al., "Mass and Energy Analysis and Space-Resolved Measurements of Ions from Plasma Focus Devices", Phys. Lett., 79A, 5, (1980).

[12] M. J. Rhee, "Heavy-ion beams produced by high-voltage pulse-powered plasma focus", Appl. Phys. Lett., 37, 10, 906, (1980).

[13] Y. Kondoh, K. Shimoda, and K. Hirano, "Measurements of Energetic Particle Beams in a Plasma Focus", J. Journal App. Phys., 20, 2, 393 (1981).

[14] I. Lindemuth and B. Freeman, "Shock dynamics and neutron production in an explosive generator driven dense plasma focus", Appl. Phys. Lett., 40, 6, 462, (1982).

[15] W. Stygar, G. Gerdin, F. Venneri, and J. Mandrekas, "Particle Beams Generated by a 6-12.5 kJ Dense Plasma Focus", Nucl. Fusion, 22, 9, (1982).

[16] T. Yamamoto, Y. Kondoh, K. Shimoda, and K. Hirano, "Measurement of Electron- and Ion Beam Energies and Currents in a Plasma Focus Discharge", J. Jornal App. Phys., 21, 4, 659, (1982).

[17] M. Tanimoto, I. Ueno, K. Koyama and Y. Matsumoto, "Comparative Study of Various Models of Energetic Ion Generation from Plasma Focus”, J. Journal App. Phys., 23, 11, 1470, (1984).

[18] T. Yamamoto, K. Shimoda, and K. Hirano, "Neutrons, X-Rays and Charged Particle Beams Emission in a $65 \mathrm{kV}$ Plasma Focus”, J. Journal App. Phys., 24, 3, 324, (1985).

[19] Y. Kondoh and M. Mamada, "Numerical study on charged particle accelerations in the plasma focus", Phys. Fluids, 29, 2, 483, (1986).

[20] U. Jager and H. Herold, "Fast Ion Kinetics and Fusion Reaction Mechanism in the Plasma Focus", Nucl. Fusion, 27, 3, (1987).

[21] R. Deutsch and W. Kies, "Ion acceleration and runaway in dynamical pinches”, Plasma Phys. Cont. Fusion, 30, 3, 263, (1988).

[22] M. Sadowski, J. Zebrowski, E. Rydygier, and J. Kucinski, "Ion Emission from Plasma-Focus Facilities”, Plasma Phys. Cont. Fusion, 30, 6, 763, (1988).

[23] I. Tiseanu, N. Mandache, and V. Zambreanu, "Energetic and angular characteristics of the reacting deuterons in a plasma focus", Plasma Phys. Cont. Fusion, 36, 417, (1994).

[24] H. Kelly and A. Marquez, "Ion-beam and neutron production in a low-energy plasma focus", Plasma Phys. Cont. Fusion, 38, 1931, (1996).

[25] M. Zakaullah, I. Akhtar, A. Waheed et al., "Comparative study of ion, x-ray and neutron emission in a low energy plasma foucs”, Plasma Sources Sci. Technol., 7, 206, (1998). 
[26] S. Springham, S. Lee, and M. Rafique, "Correlated deuteron energy spectra and neutron yield for a $3 \mathrm{~kJ}$ plasma focus", Plasma Phys. Cont. Fusion, 42, 1023, (2000).

[27] M. Milanese, R. Moroso, and J. Pouzo, "D-D neutron yield in the $125 \mathrm{~J}$ dense plasma focus Nanofocus", Eur. Phys. J. D 27,77, (2003).

[28] L. Rapezzi, M. Angelone, M. Pillon et al., "Development of a mobile and repetitive plasma focus", Plasma Sources Sci. Technol., 13, 272, (2004).

[29] M. Scholz, B. Bienkowska, I. Ivanova-Stanik et al., "General characteristics of fusion-neutron emission from megajoule plasma-focus facility”, Czec. J. Phys., 56, B243, (2006).

[30] H. Yousefi, S. Mohanty, Y. Nakada, H. Ito, and K. Masugata, "Compression and neutron and ion beams emission mechanisms within a plasma focus device”, Phys. Plasmas, 13, 114506, (2006).

[31] V. Gribkov, A. Banaszak, B. Bienkowska, et al., "Plasma dynamics in the PF-1000 device under full-scale energy storage: II. Fast electron and ion characteristics versus neutron emission parameters and gun optimization perspectives", J. Phys. D, 40, 3592, (2007).

[32] Y. Mizuguchi, J. Sakai, H. R. Yousefi et al., "Simulation of high-energy proton production by fast magnetosonic shock waves in pinched plasma discharges", Phys. Plasmas, 14, 032704, (2007).

[33] C. Moreno, H. Bruzzone, J. Martinez, and A. Clausse, "Conceptual Engineering of Plasma-Focus Thermonuclear Pulsors", IEEE Trans. Plasma. Sci., 28, 5, 1735, (2000).

[34] J. Gonzalez, A. Clausse, H. Bruzzone, P. Florido, "A Lumped Parameter Model of Plasma Focus", IEEE Trans. Plasma Sci., 32, 3, 1383, (2004).

[35] T. Zhang, R. Rawat, S. Hassan et al., "Drive Parameter as a Design Consideration for Mather and Filippov Types of Plasma Focus", IEEE Trans. Plasma Sci., 34, 5, 2356, (2006).

[36] D. Potter, "Numerical Studies of the Plasma Focus", Phys. Fluids, 14, 9, (1971).

[37] M. Trunk, "Numerical Parameter Studies for the Dense Plasma Focus", Plasma Phys., 17, 237, (1975).

[38] S. Maxon and J. Eddleman, "Two-dimensional magnetohydrodynamic calculations of the plasma focus", Phys. Fluids 21, 10, 1856, (1978).

[39] P. Eltgroth, "Comparison of plasma focus calculations", Phys. Fluids, 25, 12, 2408, (1982).

[40] K. Behler and H. Bruhns, "Three-fluid magnetohydrodynamical simulation of plasma focus discharges", Phys. Fluids, 30, 12, 3767, (1987).

[41] W. Stepniewski, "MHD numerical modeling of the plasma focus phenomena", Vacuum, 76, 51, (2004).

[42] F. Casanova, C. Moreno, and A. Clasusse, "Finite-elements numerical model of the current-sheet movement and shaping in coaxial discharges", Plasma Phys. Cont. Fusion, 47, 1239, (2005). 